\title{
Development of Oral Dissolving Gelatin Beads Containing Allopurinol for the Prevention and Treatment of Mucositis
}

\author{
Yoshifumi Murata $^{1 *}$, Takashi Isobe ${ }^{1}$, Kyoko Kofuji $^{1}$, Masafumi Mizutani $^{2}$, Ryosei Kamaguchi ${ }^{2}$ \\ ${ }^{1}$ Faculty of Pharmaceutical Science, Hokuriku University, Kanazawa, Japan; ${ }^{2}$ Morishita Jintan Co. Osaka Technocenter, Hirakata, \\ Japan. \\ Email: *y-murata@hokuriku-u.ac.jp
}

Received March $14^{\text {th }}, 2012$; revised April 20 $0^{\text {th }}, 2012$; accepted May 26 $6^{\text {th }}, 2012$

\begin{abstract}
Oral dissolving gelatin beads (GBs) containing allopurinol (AP) were prepared by the seamless capsule method and their rheological properties were examined. The release profiles of both gelatin and AP from GBs were also investigated in limited dissolution medium. GBs containing AP provided an easy-to-handle dosage form, but the physical strength of the beads immediately decreased upon contact with physiological saline at $37^{\circ} \mathrm{C}$. Gelatin was released from the outer layer of GBs in physiological saline, with almost all the gelatin dissolved after 5 min, together with approximately $30 \%$ of the AP contained in the inner layer of the GB. The oral administration of GBs likely results in immediate softening of the GB upon contact with saliva. The released AP acts directly at inflammation sites, in a manner similar following oral rinsing with an AP suspension. Therefore, GBs are a useful dosage form for preventing or treating localized problems in the oral cavity, such as mucositis.
\end{abstract}

Keywords: Gelatin Bead; Seamless Capsule; Oral Mucositis; Allopurinol

\section{Introduction}

Oral disintegration dosage forms such as oral disintegration tablets are currently widely utilized, especially by the elderly or patients who have difficulty swallowing regular tablets [1-3]. The active compound contained in the form spreads rapidly throughout the oral cavity as the form disintegrates upon contact with saliva. Therefore, such forms are ideal for oral care if the drug acts on the oral mucosa, tongue, teeth or gums. Such oral disintegration tablets should consist of water-soluble materials since insoluble components would remain on or around the tongue. Fast dissolving films are thus more useful than tablets for releasing a drug quickly in the oral cavity $[4,5]$. However, both the amount and type of drug incurporated in the film dosage form are restricted as the drug loading capacity of film is typically very low. Soft capsules have also been successfully used as an oral dosage form [6-8]. The capsule delivers the drug solution in a solid dosage form, and is conventionally used to encapsulate liquid compounds. Soft capsules mask the unpleasant taste or smell of the drug, and are easy to swallow. Gelatin is the most popular material for the preparation of soft capsules. The disintegration of soft gelatin capsules in the gastrointestinal cavity is controlled by the type of gelatin, and the method used to prepare the gelatin gel [9].

"Corresponding author.
The objective of this work was to prepare oral dissolving gelatin beads (GBs) using the seamless capsule method [10]. A xanthine oxidase inhibitor, allopurinol (AP), was selected as the model drug for incorporation into the GBs. AP is used for the prevention and treatment of oral mucositis (mouth ulcers) which occur in cancer patients receiving chemotherapy and/or radiotherapy [11]. Treatment of mucositis requires the direct application of AP to the oral mucosa in order to avoid the systemic action that appears after gastrointestinal absorption. For example, an AP suspension $(1 \mathrm{mg} / \mathrm{mL})$ is freshly prepared in the hospital and used by the patient to rinse their oral cavity [12]. Although this method for preventing mucositis is simple and effective, the preparation is not stable. Furthermore, it is difficult to control the amount of AP rinse used by the patient. AP incorporated in a fast dissolving form should be active following dissolution in saliva upon oral administration. In this study, the rheological properties of GBs were examined in an aqueous solution, and the release profiles of both gelatin and AP from GBs were investigated in limited dissolution medium.

\section{Experimental}

\subsection{Materials}

Gelatin was obtained from Nippi Inc. (Tokyo, Japan) and 
D-sorbitol was obtained from Ogawa Co. (Tokyo, Japan). Glycerin and medium-chain triglyceride (MCT) were from Kao Co. (Tokyo, Japan). Polyethylene glycol 400 (PEG400) was from NOF Co. (Tokyo, Japan). Sucrose acetate isobutylate (SAIB) was from KOYO MERCANTILE Co. (Tokyo, Japan). AP was purchased from Wako Pure Chemicals (Osaka, Japan) and a BCA protein assay reagent kit was purchased from Thermo Fisher Scientific K.K. (Yokohama, Japan). All other chemicals were of reagent grade.

\subsection{GB Preparation}

GBs with entrapped AP were prepared by the seamless capsule method, as shown in Figure 1 [13]. Table 1 shows the composition of the base solutions (A, B, C), and other conditions for the preparation of GBs. GBs were immediately formed when the three solutions (A, B, C) were simultaneously injected through a triple nozzle into the carrier solution, $\mathrm{MCT}$, at $0^{\circ} \mathrm{C}-10^{\circ} \mathrm{C}$. The $\mathrm{GBs}$ were carried by the flow of MCT the entire length of the apparatus and were then filtered. The GBs were dried under ventilation at room temperature and were preserved in aluminum laminate pouches at $4^{\circ} \mathrm{C}$. After one week, the GBs were brought to room temperature (ca. $20^{\circ} \mathrm{C}$ ) and their diameters measured using a micrometer (CLM1-15QM; Mitutoyo, Kawasaki, Japan) at a pressure of $0.5 \mathrm{~N}$. The weights of the GBs were measured using an electric balance (CP224S; Sartorius K.K., Goettingen, Germany). Each measurement was made on twenty GBs and the mean $( \pm$ S.D.) was calculated. Both the diameter and weight of GBs stored at $4^{\circ} \mathrm{C}$ for 6 months were measured by the same methods.

\subsection{Determination of the Amount of $A P$ in the GBs}

The amount of AP contained in the GBs was determined as follows. A single GB was placed in a screw cap vial and $10 \mathrm{~mL}$ of physiological saline was added. The vial

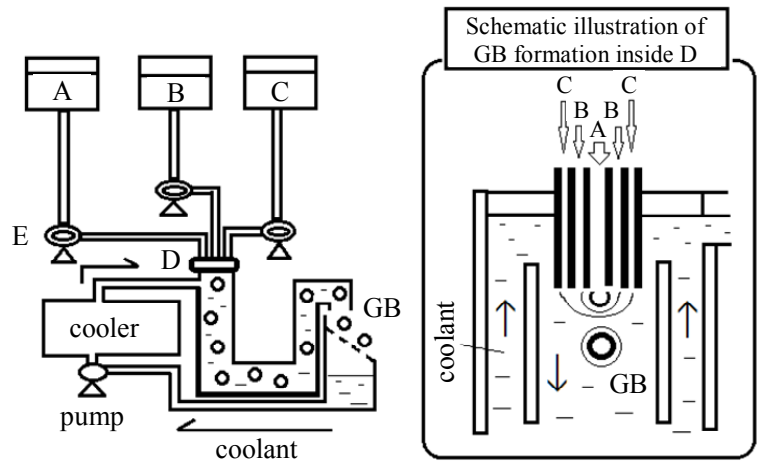

A: AP solution, B:Inner layer solution, C: Outer layer solution

D: Triple nozzle, E: Constant rate pump

Figure 1. Equipment for the preparation of GBs.
Table 1. Composition of the base solutions used for the preparation of GBs.

\begin{tabular}{cccc}
\hline Solution & $\begin{array}{c}\text { Composition } \\
(\text { weight ratio) }\end{array}$ & $\begin{array}{c}\text { Temperature } \\
\left({ }^{\circ} \mathrm{C}\right)\end{array}$ & $\begin{array}{c}\text { Flow rate } \\
(\mathrm{mL} / \mathrm{min})\end{array}$ \\
\hline A & AP:PEG400 (12:88) & ambient & 26.0 \\
B & SAIB:MCT (80:20) & 80 & 8.8 \\
C & $\begin{array}{c}\text { Gelatin:D-Sorbitol: } \\
\text { Glycerin (80:15:5) }\end{array}$ & 60 & 19.9 \\
\hline
\end{tabular}

was shaken at $300 \mathrm{rpm}$ in a shaker incubator (AS ONE Co. SI-300, Osaka, Japan) at $37^{\circ} \mathrm{C}$. After $24 \mathrm{~h}$, an $80 \mu \mathrm{L}$ aliquot was removed, placed in a micro test tube $(1.5 \mathrm{~mL})$ and $720 \mu \mathrm{L}$ of methanol was added to precipitate the gelatin dissolved from the GB. The sample was mixed and centrifuged $(7700 \times \mathrm{g}, 5$ min; Kokusan Co. H-1300, Saitama, Japan), then the supernatant was injected into an HPLC column. The HPLC system consisted of an LC-6A pump (Shimadzu Co., Kyoto, Japan), a packed column $\left(150 \mathrm{~mm} \times 4.6 \mathrm{~mm}\right.$, Cosmosil $5 \mathrm{C}_{18}$-MS-II, Nacalai Tesque, Kyoto, Japan), and a SPD-6A UV detector (Shimadzu Co.). HPLC for determining the concentration of AP was conducted at ambient temperature using $20 \mathrm{mM}$ acetate buffer ( $\mathrm{pH} 4.5)$ at a flow rate of 1.0 $\mathrm{mL} / \mathrm{min}$ [14]. The detector wavelength was set as $254 \mathrm{~nm}$. All release tests were performed in triplicate.

\subsection{Rheological Tests on GBs}

The load weight $(\mathrm{g})$ was measured with a rheometer (SUN RHEO TEX SD-700\#; Sun Scientific Co., Tokyo, Japan) at room temperature on GBs pressed to a thickness of $0.5 \mathrm{~mm}$ using a flat adapter (diameter: $10 \mathrm{~mm}$ ). The change in strength of GBs was also investigated as follows. A single GB was immersed in $10 \mathrm{ml}$ of physiological saline at $37^{\circ} \mathrm{C}$, and removed from the solution after 5, 10 or $20 \mathrm{~s}$. Water drops adhering to the GB surface were removed with a filter paper, then the rheological properties of the GB was rapidly tested as above. All tests were performed on ten GBs and the mean $( \pm$ S.D.) was calculated.

\subsection{Dissolution of Gelatin from GBs}

Physiological saline or deionized water was used as the dissolution test medium. A single GB was placed in a plastic dish (diameter, $54 \mathrm{~mm}$ ) and $10 \mathrm{~mL}$ of dissolution medium incubated at $37^{\circ} \mathrm{C}$ was added. The dish was shaken at $300 \mathrm{rpm}$ in a shaker incubator at $37^{\circ} \mathrm{C}$. A $25 \mu \mathrm{L}$ aliquot was removed periodically, placed in a microplate (96 wells), and the amount of gelatin was measured using a BCA protein assay kit in a multi-plate reader (Viento, Dainippon Seiyaku, Suita, Japan). All dissolution tests were performed in triplicate. 


\subsection{Release of AP from GBs}

Physiological saline or deionized water was used as the dissolution test medium. A single GB was placed in a plastic dish and $10 \mathrm{~mL}$ of dissolution medium incubated at $37^{\circ} \mathrm{C}$ was added. The dish was shaken at $300 \mathrm{rpm}$ in a shaker incubator at $37^{\circ} \mathrm{C}$. An $80 \mu \mathrm{L}$ aliquot was removed periodically, placed in a micro test tube, and $720 \mu \mathrm{L}$ of methanol was added. The sample was mixed and centrifuged $(7700 \times \mathrm{g}, 5 \mathrm{~min})$, then the supernatant was injected into an HPLC column. All tests were performed in triplicate.

\section{Results and Discussions}

Homogenous GBs were readily obtained using the seamless capsule method, as shown in Figure 2. The formation of GBs was not affected by the addition of AP. The mean diameter of the GBs was $4 \mathrm{~mm}$. GBs did not adhere to the surface of the vessel or the equipment after drying, and aggregation of the GBs was not observed. The amount of AP incorporated in each GB was $3.48 \pm$ $0.16 \mathrm{mg}(\mathrm{c} . \mathrm{v} .=4.6 \%)$. GBs were stored at $4^{\circ} \mathrm{C}$ in a tight container, as gelatin gel contracts if the water in the get matrix evaporates. Both the diameter and the weight of the GBs stored under these conditions did not change over a period of 6 months, as shown in Table 2 .

Since GBs are placed into the oral cavity, they must be easy to handle. The GBs had a rigid capsule exterior and could be easily handled without deforming them. However, their rheological characteristics drastically changed upon contact with physiological saline at $37^{\circ} \mathrm{C}$. The strength of GBs containing AP decreased dramatically after 5 s, as shown in Figure 3. The GBs became soft gel

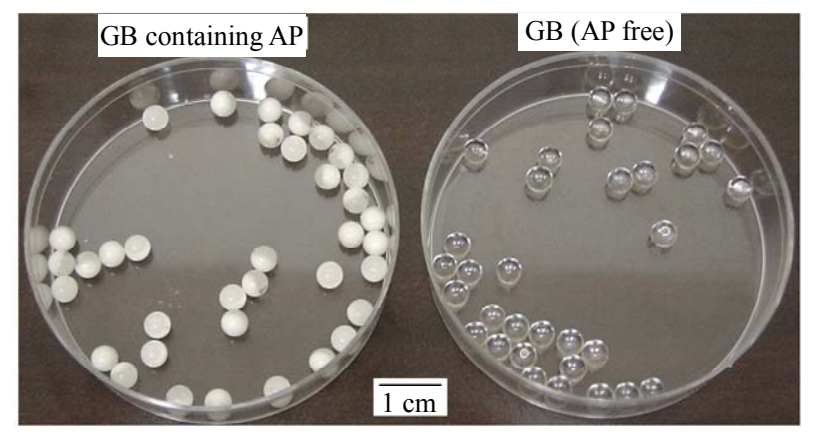

Figure 2. Photograph of GBs.

Table 2. Change in size and weight of GBs stored at $4^{\circ} \mathrm{C}$.

\begin{tabular}{lcclcc}
\hline Sample & Storage & Diameter $(\mathrm{mm})$ & \multicolumn{2}{c}{ Weight $(\mathrm{mg})$} \\
\hline GB containing AP & 1 week & 4.0 & 0.0 & 40.9 & 0.5 \\
GB containing AP & 6 months & 4.0 & 0.0 & 40.7 & 0.6 \\
GB (AP free) & 1 week & 4.0 & 0.1 & 36.7 & 2.6 \\
GB (AP free) & 6 months & 3.9 & 0.1 & 36.8 & 2.2 \\
\hline
\end{tabular}

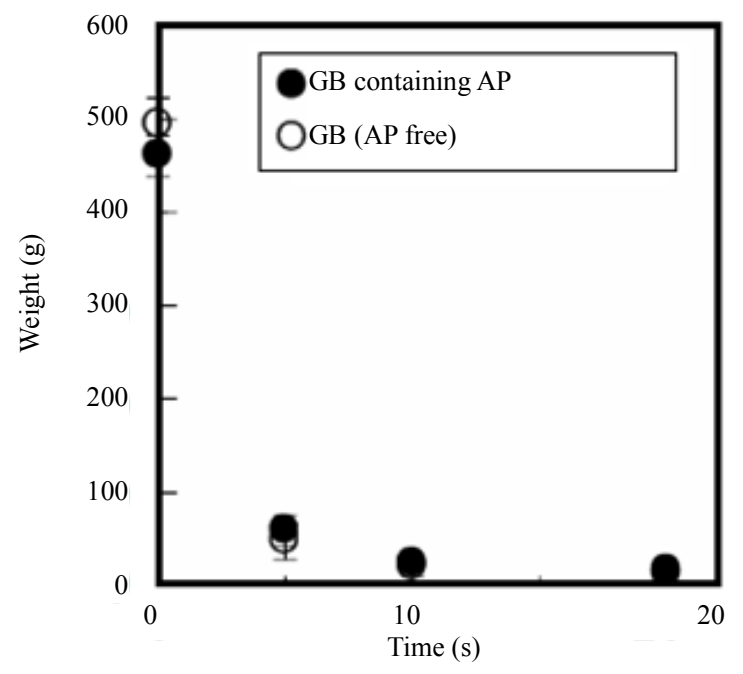

Figure 3. Change in the hardness of GBs in physiological saline at $37^{\circ} \mathrm{C}$.

beads, and could not be handled after $20 \mathrm{~s}$. This rapid softening was also observed with GBs lacking AP. This softening might be due to dissolution of gelatin from the outer layer of the GBs, since surface erosion was observed visually.

Soft capsules are a dosage form that can be erroneously swallowed. Therefore, GBs must quickly disintergrate after administration for treatment of mucositis. In this study, a single GB was soaked in $10 \mathrm{~mL}$ of test solution and the amount of gelatin released was measured. Figure 4 shows the release profile of gelatin from individual GBs. Gelatin was immediately released from GBs, whether or not they contained AP, upon immersion in physiological saline. In all cases, most of the gelatin contained in a GB dissolved after $5 \mathrm{~min}$. Similar gelatin release profiles were obtained when deionized water was used instead of saline. These results show that the gel matrix structure of GBs will disintegrate upon contact with small amounts of saliva.

There is no AP in the outer layer of GBs prepared by the present method; AP is found only in the inner layer, which consists of PEG 400. Thus, AP is released following dissolution of the gelatin. As shown in Figure 5, approximately $30 \%$ of the AP contained in the inner layer of a GB was released in physiological saline or deionized water after $5 \mathrm{~min}$, although the amount released varied widely, depending on the disintegration rate of the GB. The release rate of AP obtained in these experiments might be reasonable for the treatment of mucositis because saliva is secreted from the salivary glands at 0.3 $0.4 \mathrm{~mL} / \mathrm{min}$ under resting conditions [15].

\section{Conclusion}

In this study, GBs containing AP and designed to disintegrate in the oral cavity were prepared using the seam- 

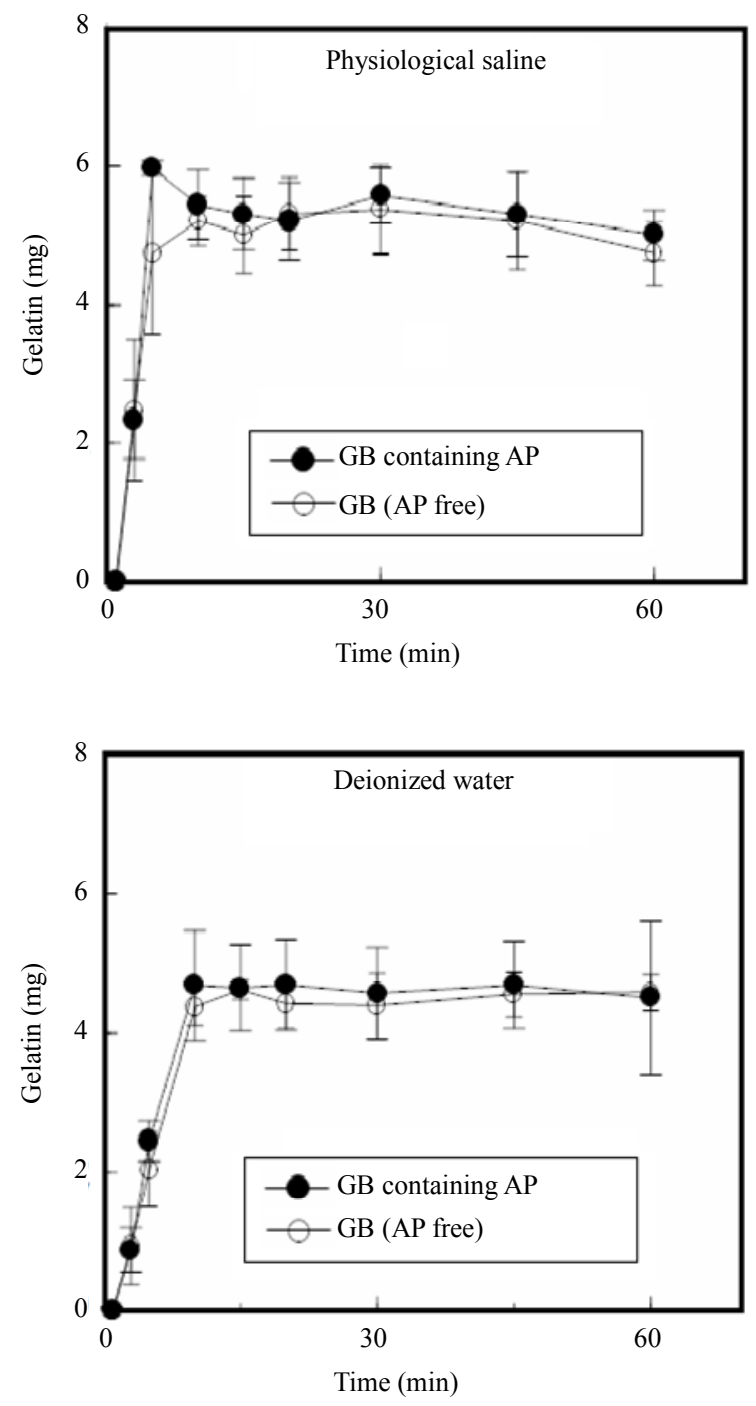

Figure 4. Release profiles of gelatin from GBs in dissolution medium.

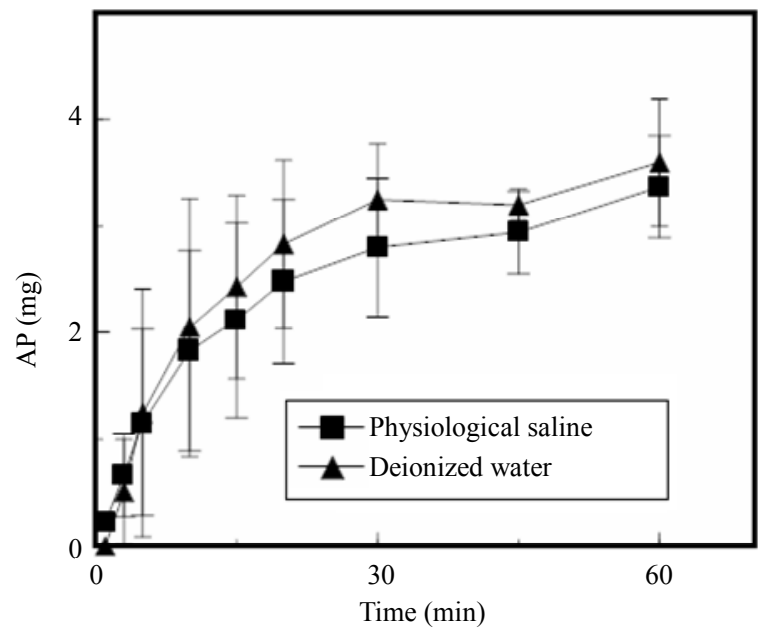

Figure 5. Release profiles of AP from GBs in dissolution medium. less capsule method. The gelatin immediately dissolved and AP incorporated in the GBs was released into limited dissolution medium. When GBs are orally administered, the outer layer softens upon contact with saliva and AP contained in the inner layer is released. AP dissolved in saliva acts directly at the inflammation site in a manner similar to that of AP suspension administered as an oral rinse. Therefore, GBs are a useful dosage form for preventing or treating localized problems in the oral cavity, such as mucositis. It is also a valuable form when a person in attendance must administer the drug to a cancer patient receiving chemotherapy and/or radiotherapy. Since the size and properties of GBs can be controlled by the conditions used for preparing GBs, we are now investigating the oral dissolution of GB in vivo.

\section{REFERENCES}

[1] J. Breitkreutz and J. Boos, "Drug Delivery and Formulations," Handbook of Experimental Pharmacology, Vol. 205, 2011, pp. 91-107.

[2] V. Parkash, S. Maan, Deepika, S. K. Yadav, Hemlata and V. Jogpal, "Fast Disintegrating Tablets: Opportunity in Drug Delivery System," Journal of Advanced Pharmaceutical Technology \& Research, Vol. 2, No. 4, 2011, pp. 223-235.

[3] S. Nalamachu, D. Hassman, M. S. Wallace, S. Dumble, R. Derrick and J. Howell, "Long-Term Effectiveness and Tolerability of Sublingual Fentanyl Orally Disintegrating Tablet for the Treatment of Breakthrough Cancer Pain," Current Medical Research and Opinion, Vol. 27, No. 3, 2011, pp. 519-530. doi:10.1185/03007995.2010.545380

[4] F. Cilurzo, I. E. Cupone, P. Minghetti, S. Buratti, C. G. Gennari and L. Montanari, "Diclofenac Fast-Dissolving Film: Suppression of Bitterness by a Taste-Sensing System," Drug Development and Industrial Pharmacy, Vol. 37, No. 3, 2011, pp. 252-259. doi: $10.3109 / 03639045.2010 .505928$

[5] V. Garsuch and J. Breitkreutz, "Comparative Investigations on Different Polymers for the Preparation of FastDissolving Oral Films," Journal of Pharmacy and Pharmacology, Vol. 62, 2010, pp. 539-545.

[6] R. C. Rossi, C. L. Dias, E. M. Donato, L. A. Martins, A. M. Bergold and P. E. Fröehlich, "Development and Validation of Dissolution Test for Ritonavir Soft Gelatin Capsules Based on in Vivo Data," International Journal of Pharmaceutics, Vol. 338, No. 1-2, 2007, pp. 119-124. doi:10.1016/j.ijpharm.2007.01.036

[7] J. R. Zuniga, R. J. Noveck, W. K. Schmidt, S. E. Boesing and E. V. Hersh, "Onset of Action of Diclofenac Potassium Liquid-Filled Capsules in Dental Surgery Patients," Current Medical Research and Opinion, Vol. 27, No. 9, 2011, pp. 1733-1739. doi:10.1185/03007995.2011.600300

[8] G. Carlomagno, S. De Grazia, V. Unfer and F. Manna, "Myo-Inositol in a New Pharmaceutical Form: A Step forward to a Broader Clinical Use," Expert Opinion on 
Drug Delivery, Epub ahead of Print (2012). doi:10.1517/17425247.2012.662953

[9] R. P. Gullapalli, "Soft Gelatin Capsules (Softgels)," Journal of Pharmaceutical Sciences, Vol. 99, No. 10, 2010, pp. 4107-4148. doi:10.1002/jps.22151

[10] K. Taki, F. Takayama and T. Niwa, "Beneficial Effects of Bifidobacteria in a Gastroresistant Seamless Capsule on Hyperhomocysteinemia in Hemodialysis Patients," Journal of Renal Nutrition, Vol. 15, No. 1, 2005, pp. 77-80. doi:10.1053/j.jrn.2004.09.028

[11] D. M. Keefe, M. M. Schubert, L. S. Elting, S. T. Sonis, J. B. Epstein, J. E. Raber-Durlacher, C. A. Migliorati, D. B. McGuire, R. D. Hutchins and D. E. Peterson, "Updated Clinical Practice Guidelines for the Prevention and Treatment of Mucositis," Cancer, Vol. 109, No. 5, 2007, pp. 820-831. doi:10.1002/cncr.22484

[12] H. Dozono, M. Murakami, T. Watanabe, Y. Nagata, K. Nakamura, M. Ishibashi and K. Yokoi, "The Prevention of Stomatitis Induced by Anti-Cancer Drugs," Gan to Kagaku Ryoho, Vol. 17, 1990, pp. 931-933.

[13] M. Asada, R. Kamaguchi, T. Ohno, T. Kozaki and T. Sugimoto, "Seamless Capsule Entrapped Living Microorganisms," Seibutsu-kogaku, Vol. 87, No. 3, 2009, pp. 123-128.

[14] M. K. Reinders, L. C. Nijdam, E. N. van Roon, K. L. Movig, T. L. Jansen, M. A. van de Laar and J. Brouwers, "A Simple Method for Quantification of Allopurinol and Oxipurinol in Human Serum by High-Performance Liquid Chromatography with UV-Detection," Journal of Pharmaceutical and Biomedical Analysis, Vol. 45, No. 2, 2007, pp. 312-317. doi:10.1016/j.jpba.2007.08.002

[15] C. Dawes, "Physiological Factors Affecting Salivary Flow Rate, Oral Sugar Clearance, and the Sensation of Dry Mouth in Man," Journal of Dental Research, Vol. 66, 1987, pp. 648-653. 